\title{
ECG Signal Denoising Based on Improved MP Algorithm
}

\author{
Wei A-ying ${ }^{1}$, Guo Yin-jing ${ }^{1}$ and Cheng Xue-zhen ${ }^{2}$ \\ ${ }^{1}$ College of electronic communication and physical, Shandong University of \\ Science and Technology, Qingdao, 266590, China \\ ${ }^{2}$ College of electrical and automation, Shandong University of Science and \\ Technology, Qingdao, 266590, China \\ greenfox66@163.com
}

\begin{abstract}
The electrocardiogram (ECG) signal processing plays an important role in diagnosing cardiopathy. Sparse decomposition provides a new tool for ECG signal processing. Matching pursuit (MP) algorithm uses overcomplete dictionary to decompose the signal, so it can reflect the properties of the signal. In decomposition process, searching a best atom of the dictionary is an optimal problem; Genetic Algorithm (GA) is used to solve this problem. This paper proposed an improved MP algorithm based on GA to denoise the ECG signal, whose dictionary is composed of Gabor atoms. The experiment simulation results show that the proposed method can get a good denosing effect in a shorter time than not use GA algorithm.
\end{abstract}

Keywords: ECG denosing, Gabor dictionary, MP, Genetic Algorithm(GA)

\section{Introduction}

Cardiopathy is one of the major diseases of human. The ECG (Electro-Cardio-Gram), which shows the Electrophysiological activity in the heart, is a nondestructive, safe, essential tool for diagnosing of cardiopathy.It is crucial for doctor to diagnose the cardiac diseases accurately by analyzing of ECG signals. So ECG signal processing plays an important role in the medical signal processing. Among these signal processing, signal denosing is one important part. Because ECG signal is collected from the body surface through the cadioelectric leads, it is very easy to be interfered by instrument and body [1]. These interferences are called noise. The main noises sources in the ECG recordings are: (1) power line interferences, with frequency being $50 \mathrm{~Hz},(2)$ muscle electricity noise, which caused by muscle activity, and (3) baseline wander, which caused by respiration or the myocardial excitation. The noises make it difficult for us to analyze and diagnosis the real signal. Therefore it is very important to remove these noises before the use. In literature, several classical methods have been proposed to solve the problem, such as the wavelet transform [2-6], adaptive filtering [7], and independent component analysis [8], etc.

For signal processing, the traditional processing methods for signal expression, such as functions of sina function and wavelet base, have good physical meaning and can get better expression effect for specific type signal. But these methods are all trying to express an random signal by a single, complete and the finite number basis functions, which are not flexible enough and may miss some information of the signal especially for the signal with wide range of time and frequency shift. The better signal processing solution to select the appropriate basis function adaptively according to the character of the signal.

In this paper, we denoise the ECG signal using MP algorithm which was first introduced by Mallat and Zhang in 1993 as a technique for signal processing [9], the signal can be expanded into waveforms whose time-frequency properties are adapted to 
its local structures by using the algorithm. While in the process of decomposition, searching a best atom of the dictionary is an optimal problem, In order to solve the problem and improve the speed of the process, Genetic Algorithm (GA) was used to find the best atom from the dictionary [10-11]. The final experiment results show that it can maintain the ECG characteristic information well while removing most of the noises.

The remainder of this paper is organized as follows: In Section 2 presents MP algorithm, in which we introduces how to decompose the signal, the detail of MP algorithm procedure and the detail constitute of the dictionary we used in our experiment; In Section 3, Genetic Algorithm (GA) approach is briefly mentioned; and Section 4 gives the detail of simulation experiment, we process the ECG signal using the algorithm we proposed. Then the conclusion is described in Section 5.

\section{MP Algorithm}

In order to decompose the signals more effectively, a better way is to select appropriate basis function adaptively according to the signal itself. Based on the wavelet analysis, Mallat and Zhang proposed an over-complete dictionary sparse decomposition thought, which chooses the waveforms that are best adapted to decompose the signal. The waveforms are called atoms, and the set of the waveforms is called dictionary. The result of decomposition is to get more concise expression (that is sparse expression).

\subsection{Signal Decompositions}

In the traditional signal decomposition, the signal can not be decomposed flexibly because the base function is unique. Atom decomposition is the expansion of base function. Any function $s$ can be represented by (1) based on over-complete base waveforms function dictionary.

$s=\sum_{r \in \Gamma} a_{r} \varphi_{r}=\phi a$

Or can be expressed by the similar formula (2):

$s=\sum_{i=1}^{m} a_{r_{i}} \varphi_{r_{i}}+r_{m}$

In the above expressions, ${ }^{a}$ is the decomposition coefficient; $r$ is the parameter of base waveforms function; ${ }^{\phi}$ is the family of base waveform function; ${ }^{r_{m}}$ is signal residue.

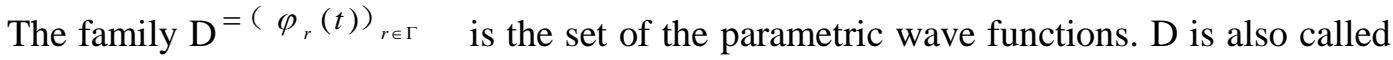
dictionary, which is redundant generally. That is to say the number of elements of the dictionary is larger than the length of the signal, and some of it can be expressed by others.

The Parametric wave functions $\left(\varphi_{r}\right)$ are the atoms of the dictionary. Different signal may have its own dictionary. That means any signal can be expressed by the superposition of a series of atoms. In order to represent any signal efficiently, an appropriate subset of atoms should be selected.

Atom decomposition should select base waveform functions to best match the signal structures from a redundant dictionary of functions. The choice may not be unique due to the redundancy of the dictionary, so the signal can be decomposed flexibly depends on the specific signal structures.

Today many dictionaries have been constructed to match the signal structures. For instance, Fourier dictionary is a popular dictionary, which is the set of Harmonic trigonometric functions and is appropriate to analyze harmonic signals; Dirac dictionary is 
the set of a series of Dirac functions and is suitable for analyzing the impulse signal; Wavelet dictionary is a typical time-scale functionary, which is built by scale expansion and translation variation.

\subsection{MP Algorithm}

Matching pursuit algorithm is an iterative greedy algorithm. The specific calculation steps are described as follows $[1,14,15]$ :

1) Initialization: let the initial residual signal is equal to the original signal, that is $r^{0} s=s$, the initial iterative time $i=0$;

2) Coefficient of inner product calculation: calculate the inner product of residual signal and all atoms of the dictionary $a_{r}=<r^{i} s, \varphi_{r \in \Gamma}>$;

3) Find the maximum of inner product $r_{i}=\arg \max \left|a_{r}\right|$;

4) Update the residual signal $r^{i+1} s=r^{i} s-a_{r_{i}} \varphi_{r_{i}}$;

5) Stop rule: give a threshold in advance, and compare the residual signal and threshold, if the residual signal is less than or equal to the threshold, then stop iteration, otherwise turn to step 2).

6) The residual signal will decrease quickly along with the decompositions. It has been proved that the residual signal will be decreased to zero exponentially. The signal can be decomposed by the equation (3) after L times:

$s=\sum_{i=0}^{\mathrm{L}-1}<r^{i} s, \varphi_{r_{i}}>\varphi_{r_{i}}+r^{i+1} s$

The procedure of MP algorithm proposed in the paper is shown in Figure 1, which is repeated until some pre-determined criteria are satisfied: 


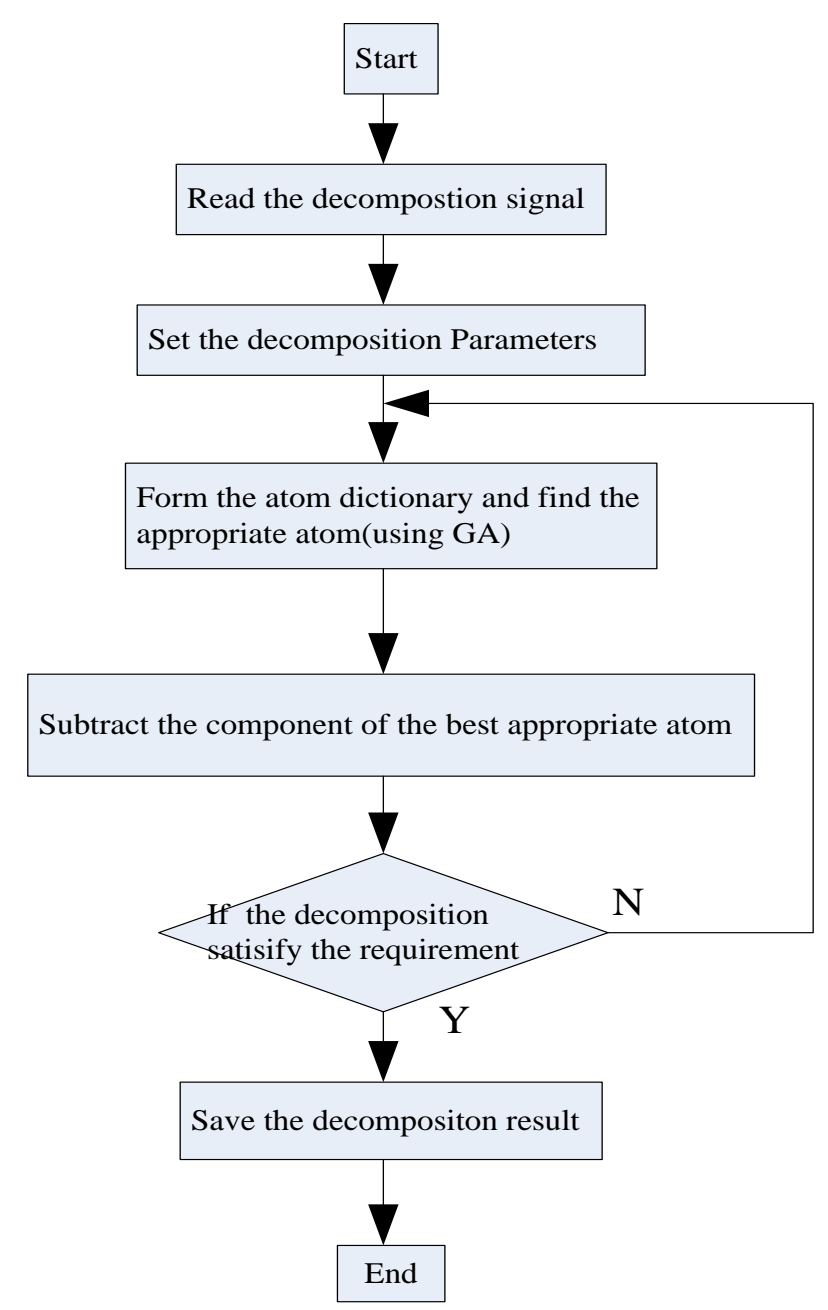

Figure 1. General Block Diagram of MP Algorithm

\subsection{Gabor Dictionary}

Because ECG is a singular signal, and Gabor atom has better expression ability for the singular signal, we used the over-complete garbor dictionary in our method [16-17]. The Gabor transform is a collection of short time Fourier transforms (STFT). The garbor dictionary is composed by the gabor atom, which is a Gaussian window defined by the (4):

$\varphi_{r}(t)=\frac{1}{\sqrt{s}} \varphi\left(\frac{t-u}{s}\right) \cos (v t+w)$

In (4), $\varphi(t)=e^{-\pi t^{2}}$ is a Gaussian function, $r=(s, u, v, w)$ is time-frequency parameters, where $\mathrm{s}$ is scale factor, $\mathrm{u}$ is displacement factor, $\mathrm{v}$ is atom frequency, and $\mathrm{w}$ is atom phase. In order to process the signal, we have to discrete the time-frequency parameters. The discrete rule is $\Gamma=\left\{\left(a^{j}, p a^{j} \Delta u, k a^{j} \Delta v, i \Delta w\right)\right\}$, where:

$$
\begin{aligned}
& a=2, \Delta u=\frac{1}{2}, \Delta v=\pi, \Delta w=\frac{\pi}{6}, 0<j<\log 2(N) \\
& 0 \leq p \leq 2^{-j+1}, 0 \leq 1 \leq 2^{j+1}, 0 \leq i \leq 12
\end{aligned}
$$




\section{Genetic Algorithm (GA)}

In MP decomposition process, searching a best atom of the dictionary is an optimal problem. The search is realized by Genetic Algorithm. It is a stochastic search method which can be used to search for an optimal solution to an optimization problem based on evolution. Genetic Algorithms (GA) introduced by John Holland in 1975 was inspired by the processes observed in natural evolution [10-13]. It simulated the breeding, crossover and mutation phenomenons occurred in the process of natural selection and natural genetic, and realized the population evolution by the selection, crossover and mutation of three basic genetic operators. In general, a population undergoes evolution: choose the fittest individuals, recombine them to generate a new population. The paper proposed GA parameter optimal approach to find a best atom from the Gabor dictionary in the process of MP decomposition. The details of our GA procedure are described as follows:

1) Initialize parameters (e.g., number of chromosomes, number of generation).

2) Create initial population randomly $(\mathrm{N})$.

3) Choose the fittest individual (compute fitness of all chromosomes).

4) evaluate population

4.1) select $N / 2$ from $N$ (parent population)

4.2) crossover

4.3) recombine new generation

5) Termination if condition satisfied, otherwise go to step 3 with $t=t+1$.

The flow chart of the genetic algorithm is shown in Figure 2.

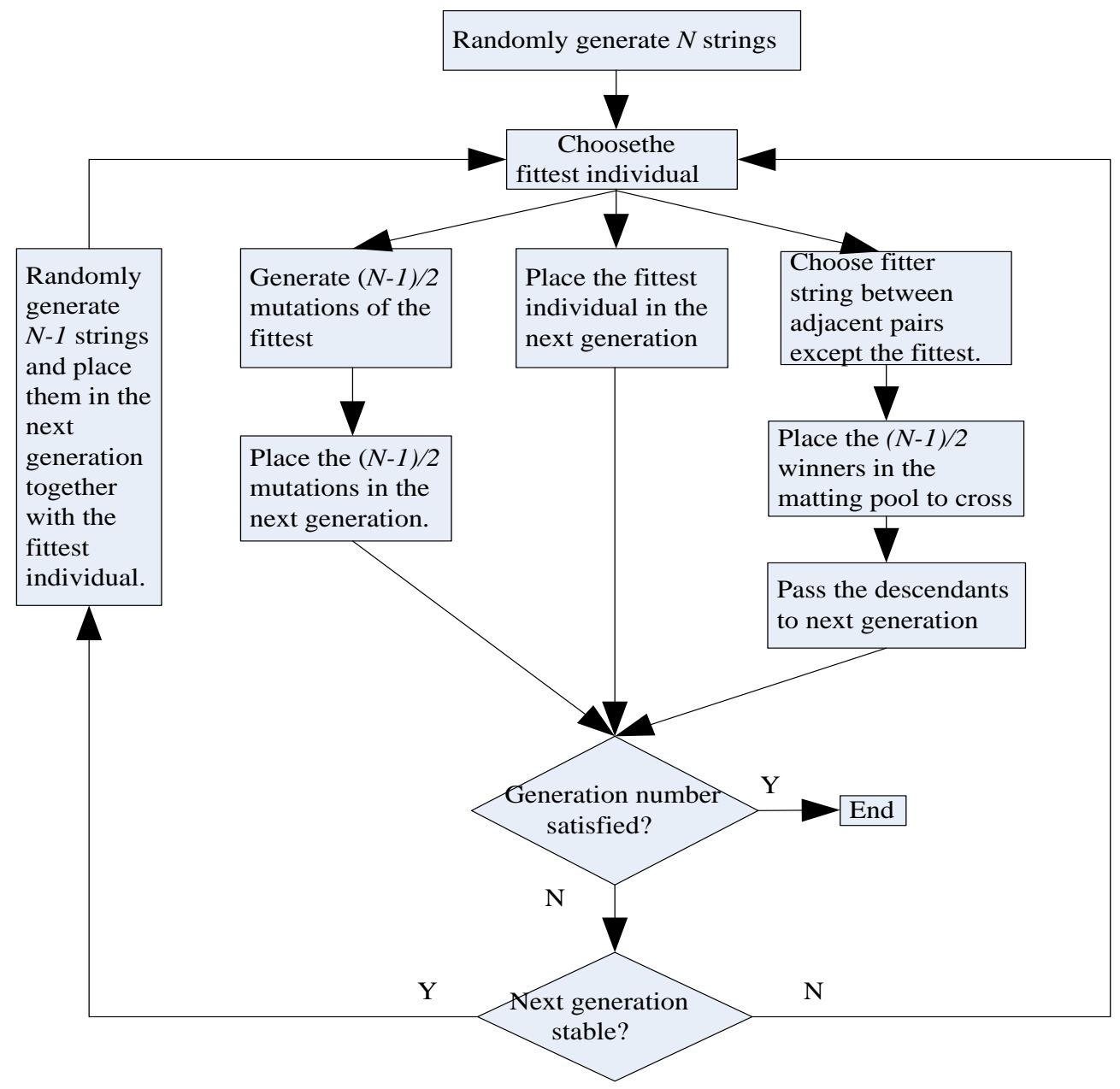

Figure 2. The Flow Chart of GA 


\section{Experiment Study}

In this section, we perform experiment to prove the performance of the MP based GA approach. The ECG signal we used in the experiment is obtained from the MIT-BIT arrhythmia database [14-15]. In order to simplify the experiment, we used the NO100 records, and saved the sample of ECG signal as text. The text is read by Matlab software, and then processed by the improved MP algorithm. The number of MP algorithm iterations is set to be 30. In the searching of the best atom, the number of Chromosomes of 21 and the number of generation of 30 are assumed. The experiment results are as follows:

Figure 3 shows the original signal with noises of NO100 record; Figure 4 shows the reconstruct signal using MP algorithm based on the GA algorithm, in which we used Garbor dictionary; Figure 5 shows one atom of the gabor dictionary; Figure 6 shows the residual signal after processing.

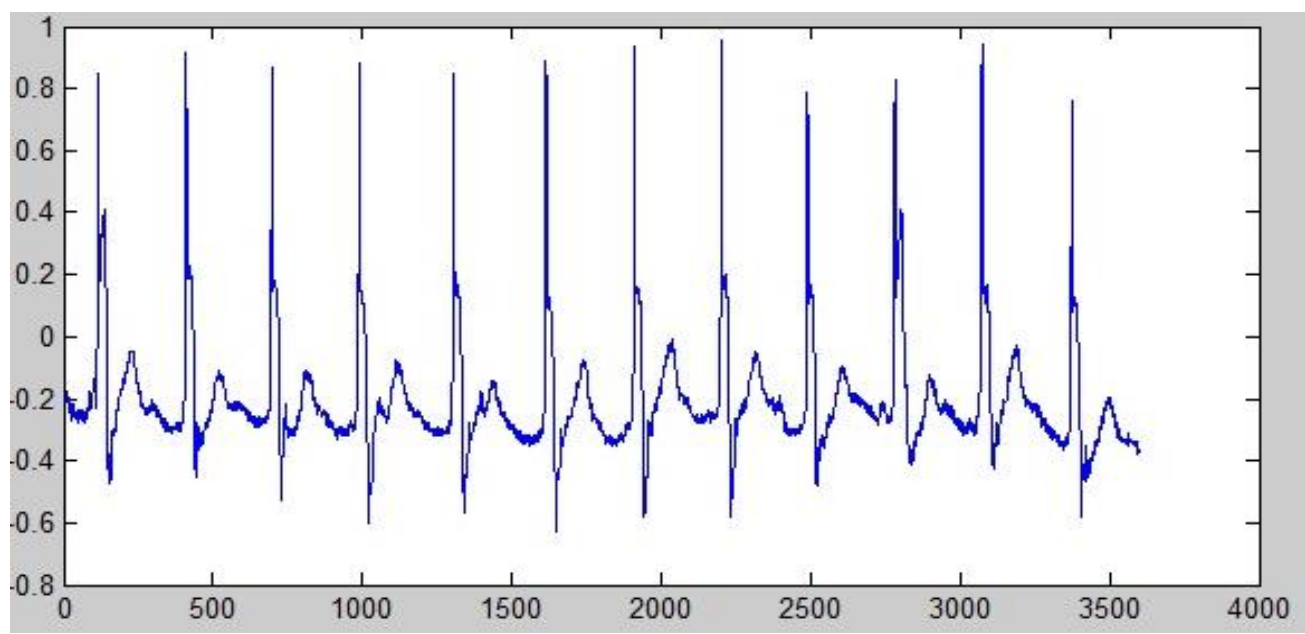

Figure 3. Original ECG Signal with Noises

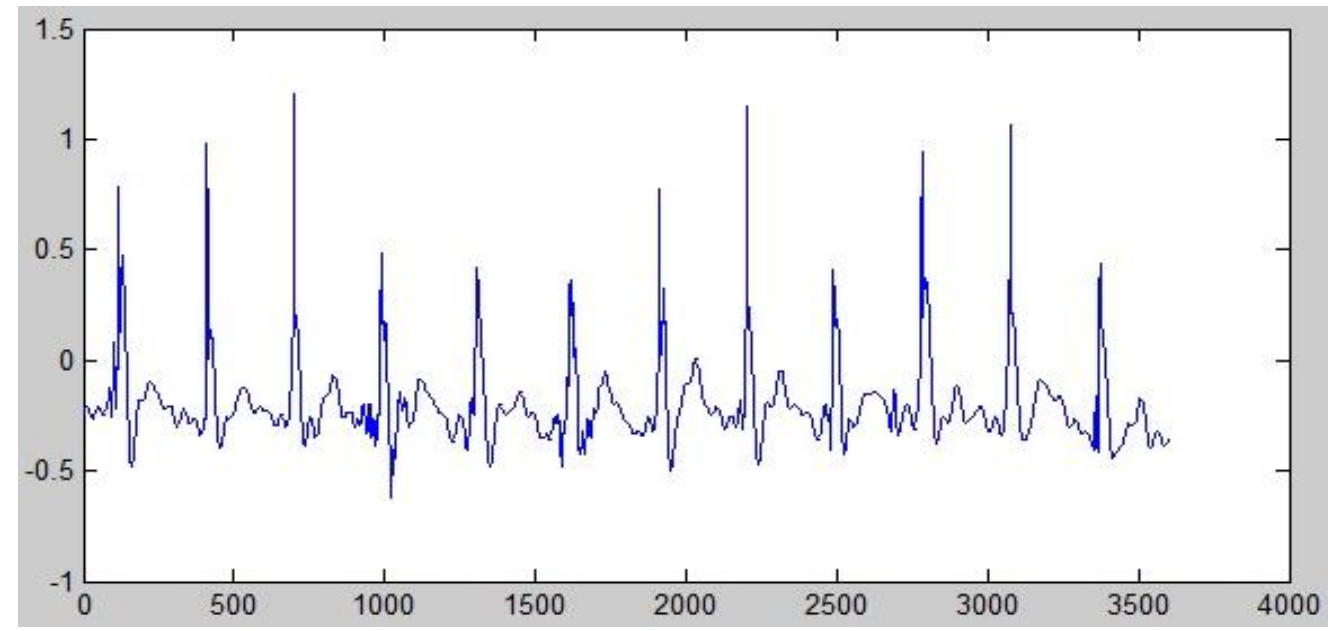

Figure 4. Reconstruct ECG Signal 


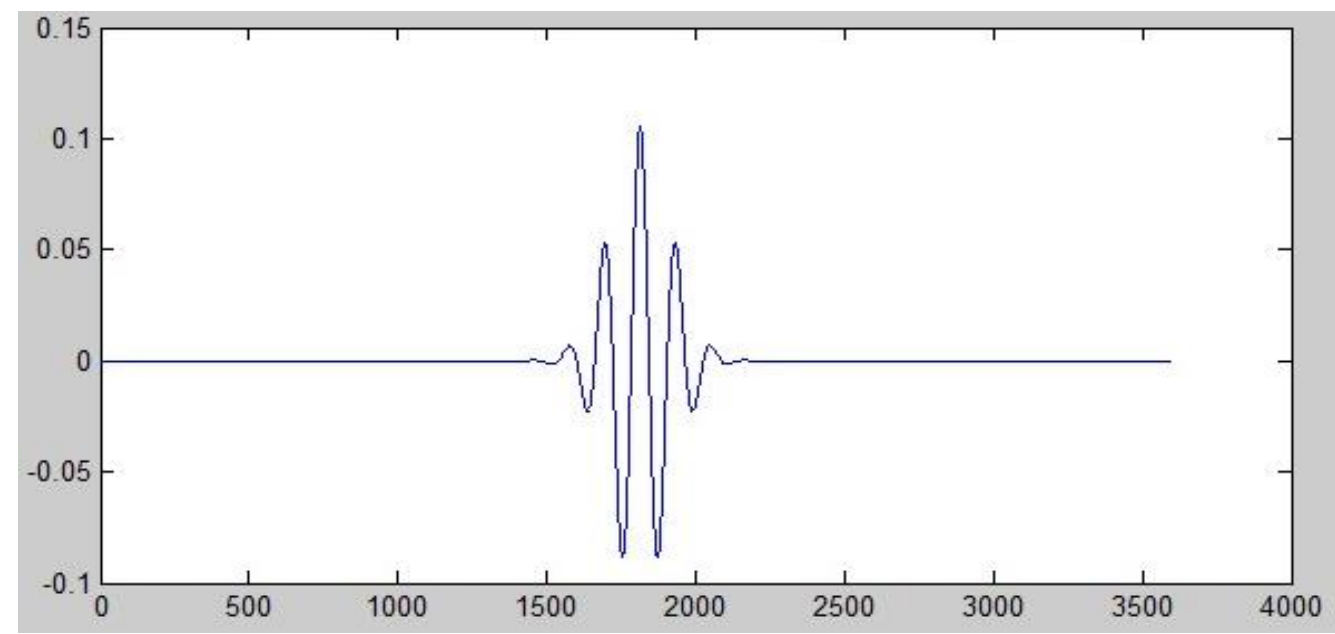

Figure 5. One of Gabor Atoms

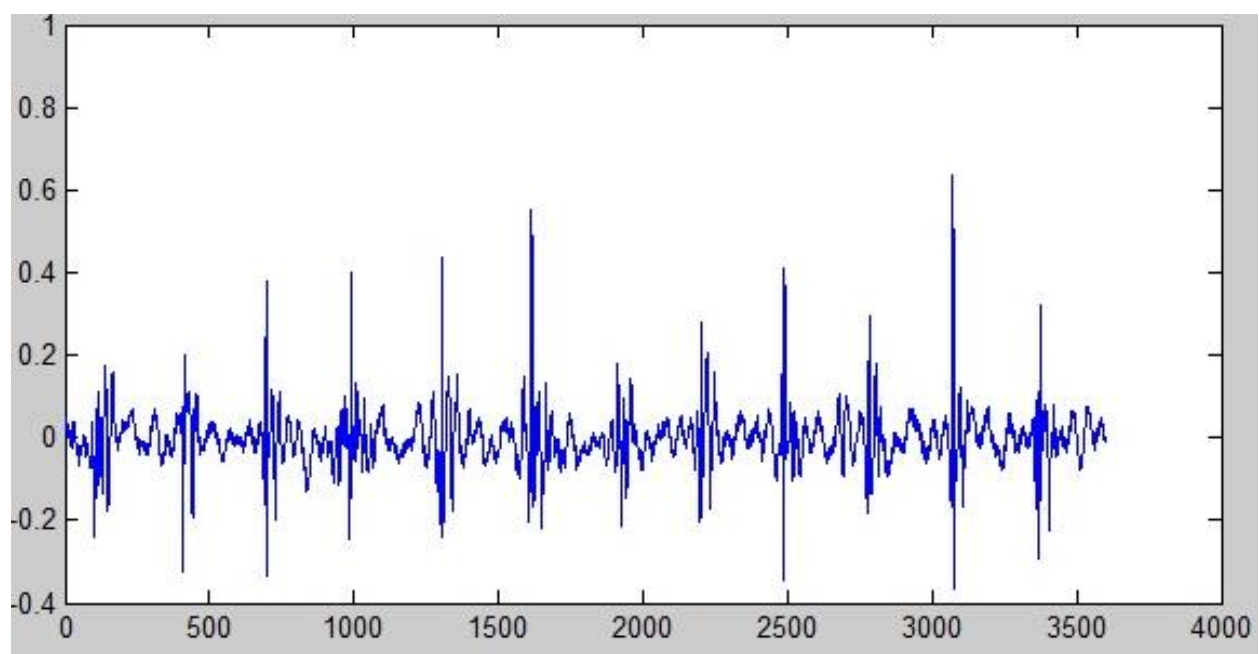

Figure 6. Residual Signal

The results of the experiment showed that the MP algorithm which used genetic algorithm had an ideal denosing effect and we can get a smoothly reconstruct ECG signal with little noises. The most important thing is that the speed of computation which used GA is improved from 90 to 100 times than the approach not used GA algorithm. The compute speed is also depend on the length of the signal, the longer length of the signal can acquire higher speed.

\section{Conclusion}

In this paper, MP algorithm has been used to denoise the ECG signals. In the decomposition procedure, most of work is spend on selecting the best atom in the dictionary suited the signal or the residual of the signal, which is an optimal problem. GA approach is considered as a global search method which can be used to search for an optimal solution to the optimal problem. Through the proper evaluation strategy, the best atom can be found from the dictionary. The experiment results based on GA algorithm showed that significant noise can be eliminated from the original signal and we can get a good reconstructed signal with most of the useful information maintained in a shorter time than the approach not used GA algorithm. In the future work, a more suitable dictionary for the MP method, which designed by the structures of the signal need to be studied. 


\section{Acknowledgments}

This work was supported by The National Natural Science Fund (No. 61471224), the Major Program of the National Natural Science Foundation of China (Grant No.U1261205), Shandong Province Large Scientific Instruments and Equipment Upgrade Program (No.2013SJGZ05).

\section{References}

[1] M. Blanco-Velasco, B. Weng and K. E. Barner, "ECG signal denoising and base line wander correction based on the empirical mode decomposition", Computers in Biology and Medicine, vol. 38, pp. 1-13, (2008). A. Alpher, J. P. N. Fotheringham-Smythe, and G. Gamow, "Can a machine frobnicate?", Journal of Foo, vol. 14, no. 1, (2004), pp.234-778.

[2] P. E. Tikkanen, "Nonlinear wavelet and wavelet packet denoising of electrocardiogram signal", Biol. Cybern., vol. 80, no. 4, (1999), pp. 259-267.

[3] C.Y.-F. Ho, B.W.-K. Ling, T.P.-L.Wong, A.Y.-P. Chan and P.K.-S. Tam, "Fuzzy multiwavelet denoising on ECG signal”, Electron. Lett., vol. 39, no.16, (2003), pp.1163-1164 .

[4] E. Ercelebi, "Electrocardiogram signals de-noising using lifting-based discrete wavelet transform", Comput. Biol. Med., vol. 34, no. 6, (2004), pp. 79-493.

[5] S. Poornachandra and N. Kumaravel, "Hyper-trim shrinkage for denoising of ECG signal", Digital Signal Process, vol. 5, no. 3, (2005), pp. 317-327.

[6] CHEN G., TANG M-h., CHENG H., and GE M., "An ECG Denoising Algorithm Based on Morphology and Wavelet Transform", Computer technology and development, vol. 22, no. 2, (2012), pp. 100-102.

[7] V. Almenar and A. Albiol, "A new adaptive scheme for ECG enhancement”, Signal Process. vol. 75, no. 3, (1999), pp. 253-26.

[8] A.K. Barros, A. Mansour and N. Ohnishi, "Removing artifacts from electrocardiographic signals using independent components analysis, Neurocomputing, vol. 22, (1998), pp. 173-186.

[9] Mallat S. and Zhang Z., "Matching pursuit with time-frequency dictionaries", IEEE Trans on Signal Processing, vol .41, no. 12, (1993), pp. 3397-3415.

[10] R. J. Vaccaro and B. F. Harrison, "Optimal Matrix_Filter design", IEEE Transactions on Signal processing, vol. 44, no. 3, (1996), pp. 705-710.

[11] Yang, S. and Jat, S. N., "Genetic Algorithms With Guided and Local Search Strategies for University Course Timetabling", IEEE Transactions on Systems, vol. 41, no. 1, (2011), pp.93-106.

[12] SUN Y-b., WU M., WEI Z-h, XIAO 1. and FENG C., "EEG spike detection using sparse representation", Acta Electronica Sinica, vol. 3, no. 9, (2009), pp. 1971-1976.

[13] JING A-, LIU Y., MA Y1., "Speech signal sparse decomposition based on matching pursuit algorithm", Computer Engineering and Applications, (2009), vol. 45, no. 5, pp.144-146.

[14] Durka PJ., "Adaptive time-frequency parametrization of epileptic spikes", Physical Review E, vol. 69, no. 05, (2004), pp.1914-1918.

[15] Durka P. J. and Blinowska KJ. A, "unified time-frequency parametrization of EEG”, IEEE Engineering in Medicineand Biology, vol. 20, no. 5, (2001), pp. 47-53.

[16] Goldberger AL, Amaral LAN, Glass L, Hausdorff JM, Ivanov PCh, Mark RG, Mietus JE, Moody GB, Peng C-K and Stanley HE., "PhysioBank, PhysioToolkit, and PhysioNet: Components of a New Research Resource for Complex Physiologic Signals", Circulation vol. 101, vol. 23, (2000), pp. e215e220, [Circulation Electronic Pages; http://circ.ahajournals.org/cgi/content/full/101/23/e215];

[17] Moody GB and Mark RG., "The impact of the MIT-BIH Arrhythmia Database", IEEE Eng in Med and Biol vol. 20, no. 3, pp. 45-50, (2001) May-June. (PMID: 11446209).

\section{Authors}

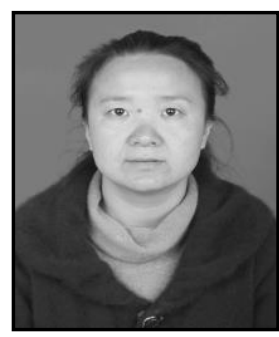

Aying wei was born in YunCheng, China in 1980. She graduated from China University of Mining And Technology in 2001. Her major research interests include signal processing, measurement technique and monitoring system. She may be reached at greenfox66@163.com. 


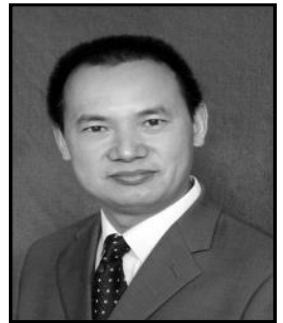

Yinjing guo was born in JiaXiang, China in 1966. He is graduated from Ordnance Engineering College of China in the year 1988. He received his master degree in 1993 and received his Ph.D degree in 2004, both from Beijing Institute of Technology (BIT). Prof. Guo's main research interests include Electromagnetic environment, Wireless communication in the complex environment and Mobile medical technology. He may be reached at GYJLWH@163.com.

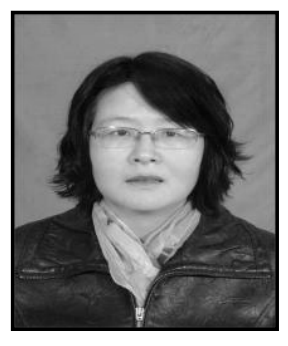

Cheng Xuezhen, was born in Linyi, China in 1964. She graduated from School of Electrical Engineering in Shandong Institute of Mining in 1987 and majored in industry electric automation. Her major research interests include signal processing, measurement technique and monitoring system. She has published over 30 papers and also has 8 patents. Professor Cheng was awarded several 2nd prize of provincial and ministerial level. Dr. Cheng may be reached at zhenxc6411@163.com. 
International Journal of Signal Processing, Image Processing and Pattern Recognition Vol.8, No.11 (2015) 